7th International Workshop on Astronomy and

Relativistic Astrophysics (IWARA 2016)

International Journal of Modern Physics: Conference Series

Vol. 45 (2017) 1760056 (5 pages)

(C) The Author(s)

DOI: $10.1142 / \mathrm{S} 2010194517600564$

\title{
QCD Phase Diagram in a Nonlocal SU(3) NJL Model with Lattice-Inspired Form Factors
}

\author{
María Florencia Izzo Villafañe, Juan Pablo Carlomagno, Daniel Gómez Dumm \\ Departamento de Física, Instituto de Física La Plata \\ Facultad de Ciencias Exactas, Universidad de La Plata (UNLP) \\ CC. 67, 1900 La Plata, Argentina \\ Consejo Nacional de Investigaciones Científicas y Técnicas (CONICET) \\ Rivadavia 1917, 1033 Buenos Aires, Argentina \\ izzo@fisica.unlp.edu.ar, carlomagno@fisica.unlp.edu.ar,dumm@fisica.unlp.edu.ar \\ Norberto N. Scoccola \\ Consejo Nacional de Investigaciones Científicas y Técnicas (CONICET) \\ Rivadavia 1917, 1033 Buenos Aires, Argentina \\ Departamento de Física, Comisión Nacional de Energía Atómica (CNEA) \\ Av. Libertador 8250, 1429 Buenos Aires, Argentina \\ Universidad Favaloro \\ Solís 453, 1078 Buenos Aires, Argentina \\ scoccola@tandar.cnea.gov.ar
}

Published 15 August 2017

\begin{abstract}
We study the QCD phase diagram in the framework of a nonlocal three-flavor quark model. We determine the model parameters from vacuum meson phenomenology, considering lattice QCD-inspired nonlocal form factors. Then we analyze the features of the deconfinement and chiral restoration transitions for systems at nonzero temperature and chemical potential.
\end{abstract}

Keywords: QCD, Phase Diagram, NJL Model.

PACS numbers: 12.38.Aw

\section{General Formalism}

We analyze the features of QCD phase transitions in the context of the so called $\mathrm{SU}(3)$ nonlocal Polyakov Nambu-Jona-Lasinio (nlPNJL) model. This is an effective theory in which quarks interact through nonlocal chirally symmetric four- and sixpoint couplings, in the presence of a background color field. The approach intends to

This is an Open Access article published by World Scientific Publishing Company. It is distributed under the terms of the Creative Commons Attribution 4.0 (CC-BY) License. Further distribution of this work is permitted, provided the original work is properly cited. 


\section{F. I. Villafañe et al.}

represent an improvement over the standard (local) PNJL model, offering a common framework to study both the chiral restoration and deconfinement transitions.

Let us start with the Euclidean effective action of the model ${ }^{1,2}$,

$$
\begin{aligned}
S_{E}= & \int d^{4} x\left\{\bar{\psi}(x)\left(-i \not D+m_{c}\right) \psi(x)-\frac{G}{2}\left[j_{a}^{s}(x) j_{a}^{s}(x)+j_{a}^{p}(x) j_{a}^{p}(x)+j^{r}(x) j^{r}(x)\right]\right. \\
& \left.-\frac{H}{4} A_{a b c}\left[j_{a}^{s}(x) j_{b}^{s}(x) j_{c}^{s}(x)-3 j_{a}^{s}(x) j_{b}^{p}(x) j_{c}^{p}(x)\right]+\mathcal{U}\left(G_{a}^{\mu}(x)\right)\right\}
\end{aligned}
$$

Here $A_{a b c}$ are SU(3) symmetric constants, while $m_{c}=\operatorname{diag}\left(m_{u}, m_{d}, m_{s}\right)$ stands for the current quark mass matrix. Assuming isospin symmetry, we take $m_{u}=m_{d}$. Quark-antiquark currents are given by

$$
j_{a}^{(s, p)}(x)=\int d^{4} z g(z) \bar{\psi}\left(x^{+}\right) \Gamma_{a}^{(s, p)} \psi\left(x^{-}\right), \quad j^{r}(x)=\int d^{4} z f(z) \bar{\psi}\left(x^{+}\right) \frac{\overleftrightarrow{i \not \partial}}{2 \kappa} \psi\left(x^{-}\right)
$$

where $x^{ \pm}=x \pm z / 2, \Gamma_{a}^{s}=\lambda_{a}, \Gamma_{a}^{p}=i \lambda_{a} \gamma_{5}$, while $g(z), f(z)$ are nonlocal form factors that characterize the effective interaction ${ }^{3}$. The action also includes an effective potential $\mathcal{U}$ that accounts for selfinteractions between color gauge fields $G_{a}^{\mu}(x)$.

We proceed by performing a standard bosonization of the theory, introducing scalar and pseudoscalar meson fields $\sigma_{a}, \zeta$ and $\pi_{a}$. Then we consider the mean field approximation (MFA), thus we expand these fields around their vacuum expectation values (VEVs). One has four nonzero VEVs, which can be written in a flavor basis as $\bar{\sigma}_{u}, \bar{\sigma}_{d}, \bar{\sigma}_{s}$ and $\bar{\zeta}$.

The bosonized action can be extended to finite temperature $T$ and chemical potential $\mu$ (we use the Matsubara formalism) to investigate the phase transitions and the behavior of the thermodynamical quantities. We assume that quarks move in a background color field

$$
\phi=i g \delta_{\mu 0} G_{a}^{\mu} \lambda_{a} / 2=\phi_{3} \lambda_{3}
$$

(Polyakov gauge), which couples to matter through the covariant derivative in the Lagrangian. After a proper regularization procedure (in which we subtract and add a free quark contribution) the mean field thermodynamical potential per unit volume is found to be given by ${ }^{2}$

$$
\Omega^{\mathrm{MFA}}=\Omega^{\mathrm{reg}}+\Omega^{\text {free }}+\mathcal{U}(\Phi, T)+\Omega_{0}
$$

where $\Omega_{0}$ is a constant, and one has

$$
\begin{aligned}
\Omega^{\mathrm{reg}}= & -2 T \sum_{n=-\infty}^{\infty} \sum_{c, f} \int \frac{d^{3} p}{(2 \pi)^{3}} \log \left[\frac{p_{n c}^{2}+M_{f}^{2}\left(p_{n c}\right)}{Z^{2}\left(p_{n c}\right)\left(p_{n c}^{2}+m_{f}^{2}\right)}\right]-\left(\bar{\zeta} \bar{R}+\frac{G}{2} \bar{R}^{2}\right. \\
& \left.+\frac{H}{4} \bar{S}_{u} \bar{S}_{d} \bar{S}_{s}\right)-\frac{1}{2} \sum_{f}\left(\bar{\sigma}_{f} \bar{S}_{f}+\frac{G}{2} \bar{S}_{f}^{2}\right)
\end{aligned}
$$




$$
\Omega^{\text {free }}=-2 T \sum_{s= \pm 1} \sum_{c, f} \int \frac{d^{3} p}{(2 \pi)^{3}} \operatorname{Re} \log \left[1+\exp \left(-\frac{\epsilon_{f p}+s\left(\mu+i \phi_{c}\right)}{T}\right)\right] .
$$

Here we have defined

$$
\begin{gathered}
p_{n c}^{2}=\left[(2 n+1) \pi T-i \mu+\phi_{c}\right]^{2}+\vec{p}^{2}, \\
Z(p)=1 /[1-\bar{\zeta} f(p)],
\end{gathered}
$$

and

$$
M_{f}(p)=Z(p)\left[m_{f}+\bar{\sigma}_{f} g(p)\right]
$$

while $\bar{S}_{f}$ and $\bar{R}$ are given by one-loop integrals that involve the scalar field VEVs ${ }^{1,2}$. The action also includes an effective pure gauge potential $\mathcal{U}(\Phi, T)$, where

$$
\Phi=\frac{1}{3}\left[1+2 \cos \left(\phi_{3} / T\right)\right]
$$

- mean field traced Polyakov loop - can be taken as order parameter of confinement. The form of the potential can be obtained from lattice QCD results and group theory constraints. We consider here both an ansatz based on the logarithmic expression of the Haar measure associated with SU(3) color group integration, as well as a widely used potential given by a polynomial based on a Ginzburg-Landau approach. The corresponding explicit forms can be found in Refs. ${ }^{4}$ and ${ }^{5}$, respectively. The potentials include a scale parameter $T_{0}$, which in presence of dynamical quarks is found to take a value of about $200 \mathrm{MeV}$ (see Ref. ${ }^{6}$ ).

The mean field values $\bar{\sigma}_{u}, \bar{\sigma}_{s}, \bar{\zeta}$ and $\phi_{3}$ (in the isospin limit, $\bar{\sigma}_{d}=\bar{\sigma}_{u}$ ) can be obtained by solving a set of four coupled "gap" equations that arise from the minimization of the mean field thermodynamical potential. Other relevant quantities are the chiral quark condensates $\langle\bar{q} q\rangle \equiv \partial \Omega^{\mathrm{MFA}} / \partial m_{q}$ and the chiral susceptibility $\chi_{c h} \equiv \partial\langle\bar{q} q\rangle / \partial m_{q}$, where $q=u, s$. For large temperatures, the behavior of the regularized quark condensates is dominated by the free contribution, which grows with $T$ as $\langle\bar{q} q\rangle \sim m_{q} T^{2}$. Therefore, in order to analyze the chiral restoration transition it is usual to define a subtracted chiral condensate, normalized to its value at $T=0$,

$$
\langle\bar{q} q\rangle_{s u b} \equiv \frac{\langle\bar{u} u\rangle-\frac{m_{u}}{m_{s}}\langle\bar{s} s\rangle}{\langle\bar{u} u\rangle_{0}-\frac{m_{u}}{m_{s}}\langle\bar{s} s\rangle_{0}} .
$$

\section{Model Parameterization}

The model includes five parameters, namely, the current quark masses $m_{u, s}$ and the coupling constants $G, H$ and $\kappa$. In addition, one has to specify the nonlocal form factors $f(z)$ and $g(z)$ entering the fermion currents. Here we will consider a parametrization chosen so as to reproduce lattice QCD results for the momentum 


\section{F. I. Villafañe et al.}

dependence of effective quark propagators. Following the analysis in Ref. ${ }^{7}$, the form factors as can be parameterized as ${ }^{8}$

$$
g(q)=\frac{1+\alpha_{z}}{1+\alpha_{z} f_{z}(q)} \frac{\alpha_{m} f_{m}(q)-m \alpha_{z} f_{z}(q)}{\alpha_{m}-m \alpha_{z}}, \quad f(q)=\frac{1+\alpha_{z}}{1+\alpha_{z} f_{z}(q)} f_{z}(q)
$$

where $\alpha_{z}=-\bar{\zeta} /(1-\bar{\zeta})$ and $\alpha_{m}=\left(m_{u}+\bar{\sigma}_{u}\right) /(1-\bar{\zeta} / \kappa)-m_{u}$, while

$$
f_{m}(q)=\left[1+\left(\frac{q^{2}}{\Lambda_{0}^{2}}\right)^{3 / 2}\right]^{-1}, \quad f_{z}(q)=\left[1+\left(\frac{q^{2}}{\Lambda_{1}^{2}}\right)\right]^{-5 / 2} .
$$

Given the form factor functions, it is possible to set the model parameters so as to reproduce the observed meson phenomenology in vacuum. Notice that the form factors introduce the cutoff scales $\Lambda_{0}$ and $\Lambda_{1}$, which have to be taken as two additional model parameters. The values of $\Lambda_{0}, \Lambda_{1}$ and $\alpha_{z}$ can be fixed from a fit to lattice QCD results for $M_{u}(p)$ and $Z(p){ }^{7}$. We obtain in this way

$$
\Lambda_{0}=861 \mathrm{MeV}, \quad \Lambda_{1}=1728 \mathrm{MeV}, \quad \alpha_{z}=-0.2492 .
$$

The remaining five parameters can be obtained by taking the value of $\alpha_{z}$ from the above fit, and by requiring that the model reproduces the empirical values of four physical input quantities. Here we take the masses of the $\pi, K$ and $\eta^{\prime}$ mesons and the pion weak decay constant $f_{\pi}$. This leads to the parameter set

$$
m_{u}=2.38 \mathrm{MeV}, m_{s}=61.45 \mathrm{MeV}, G \Lambda_{0}^{2}=14.03, H \Lambda_{0}^{5}=-158.70, \kappa / \Lambda_{0}=12.45 \text {. }
$$

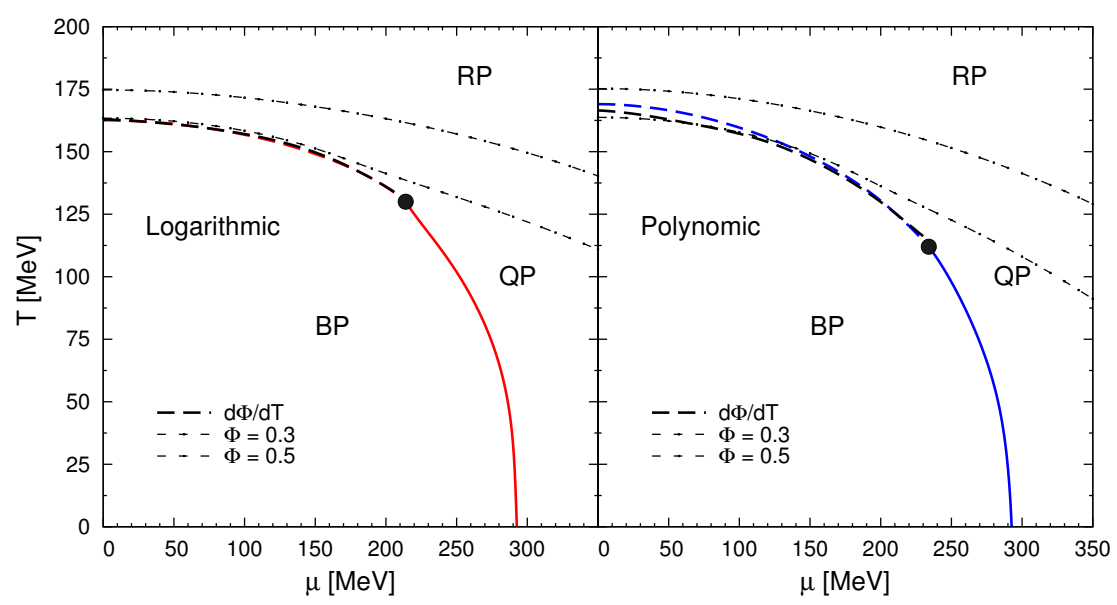

Fig. 1. Phase diagrams for logarithmic (left) and polynomial (right) PL potentials. Full (dashed) lines correspond to first order (crossover) chiral restoration transitions. Dashed-dotted lines correspond to constant $\Phi$. BP: baryonic phase, QP: quarkyonic phase, RP: restored phase. 


\section{Features of the Phase Diagram}

The phase diagram can be sketched by analyzing the numerical results obtained for the relevant order parameters. For the deconfinement and chiral symmetry restoration transitions we take as order parameters the traced Polyakov loop $\Phi$ and the subtracted chiral condensate $\langle\bar{q} q\rangle_{s u b}$, respectively. Our results are shown in Fig. 1 for both the logarithmic (left) and polynomial (right) PL potentials. At low $\mu$ we find that both transitions occur as crossovers, at about the same critical temperature $T_{c}$ for both PL potentials ( $T_{c}$ can be defined through the position of a peak in the susceptibility $\chi_{c h}$ ). By increasing $\mu$, the transition line (dashed line in Fig. 1) reaches an end point after which the chiral symmetry restoration occurs as a first order transition (solid line). Although this induces a discontinuity also in the traced Polyakov loop, it is seen that the value of $\Phi$ at the transition is relatively low. In this way, right beyond $T_{c}$ quarks are still confined, the system lying in the so-called quarkyonic phase. At some larger $T$ (for fixed $\mu$ ) quarks deconfine, however the behavior of the traced PL $\Phi$ is relatively smooth so as to find a well-establish transition line. Indicative curves for constant values $\Phi=0.3$ and $\Phi=0.5$ are shown in the figure (dashed-dotted lines).

\section{References}

1. A. Scarpettini, D. Gomez Dumm, and N.N. Scoccola, Phys. Rev. D 69, 114018 (2004).

2. J. P. Carlomagno, D. Gomez Dumm, and N. N. Scoccola, Phys. Rev. D 88, 074034 (2013).

3. G. Ripka. Quarks bound by chiral Fields, (Oxford University Press, Oxford, 1997).

4. S. Roessner, C. Ratti, and W. Weise, Phys. Rev. D 75, 034007 (2007).

5. C. Ratti, M. A. Thaler, and W. Weise, Phys. Rev. D 73, 014019 (2006).

6. B.-J. Schaefer, J. M. Pawlowski, and J. Wambach, Phys. Rev. D 76, 074023 (2007).

7. M. B. Parappilly et al., Phys. Rev. D 73, 054504 (2006).

8. S. Noguera and N. N. Scoccola, Phys. Rev. D 78, 114002 (2008). 